\title{
Bridging the ER and plasma membrane
}

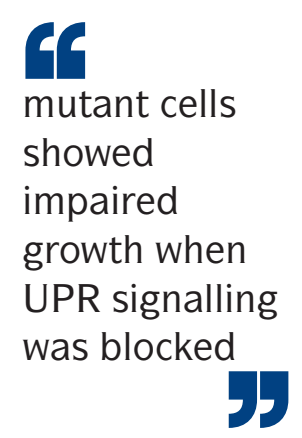

The endoplasmic reticulum (ER) forms dynamic contacts with several organelles and membranes, including the plasma membrane, and these contacts are thought to have roles in lipid transport and cell signalling. Here, Emr and colleagues characterize the structural components of ER-plasma membrane contacts in yeast and reveal their key roles in signalling and ER function.

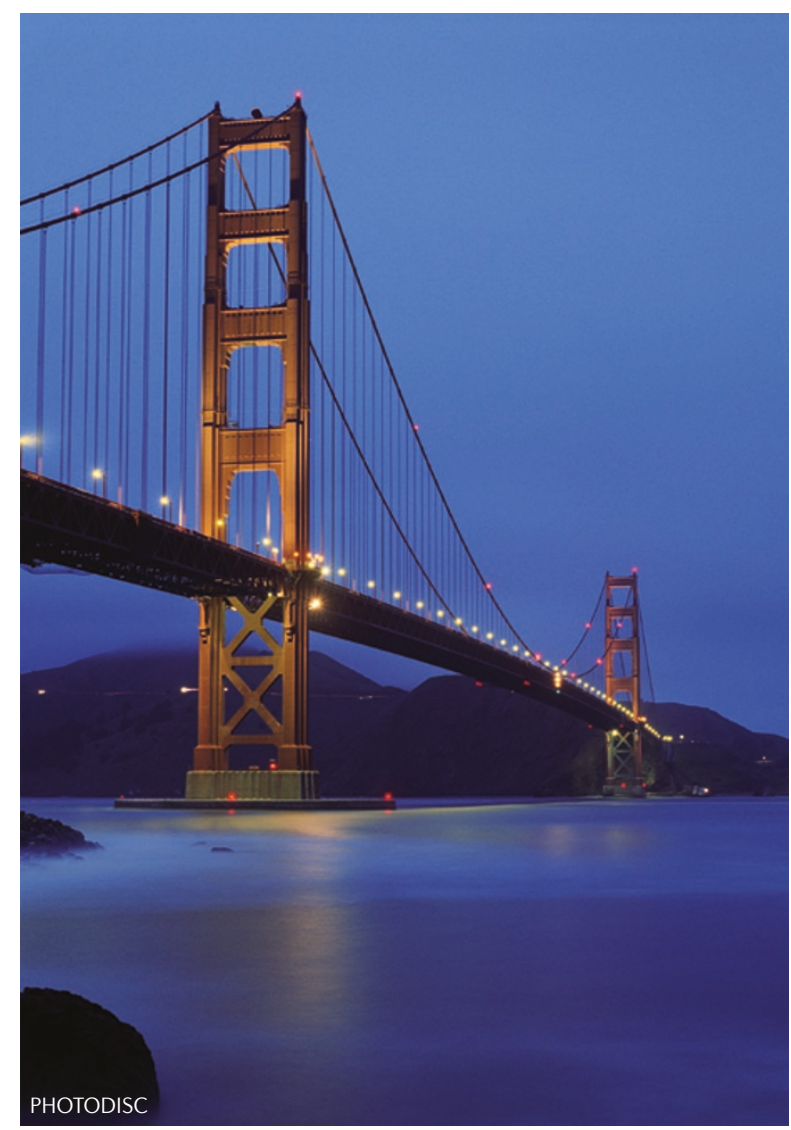

Previous work had implicated the VAMP-associated proteins (VAPs) Scs2 and Scs22 in tethering the ER to the plasma membrane in yeast, which among other functions facilitates the turnover of phosphatidylinositol-4phosphate (PtdIns4P) by the phosphatase Sac1. However, loss of Scs2 and Scs22 leads to only a modest increase in PtdIns4P levels, so the authors postulated that other proteins may be part of this tether. A search for Sac1- and Scs2-interacting proteins identified various candidates, three of which are integral membrane proteins: Ist 2 and the tricalbins Tcb1 and Tcb3.

These candidate proteins, along with Tcb2, have a protein architecture that would be expected for tether proteins. Moreover, all four proteins localized at the cortical ER (the ER network associated primarily with the plasma membrane). But do they function as ER-plasma membrane tethers? Deletion of the encoding genes, along with those coding for Scs2 and Scs22, resulted in fewer ER-plasma membrane contacts and a substantial reduction of the cortical ER. Moreover, sequential deletion of the proteins revealed a hierarchy in their roles as tethers, with Scs2 and Scs22 having a key role in initiating ER-plasma membrane junctions, followed by contributions from Ist 2 and then Tcb1, Tcb2 and Tcb3.

The authors then assessed whether loss of the four newly identified tether proteins together with Scs 2 and Scs 22 results in accumulation of PtdIns4P, and found that PtdIns4P levels were greatly increased. Furthermore, PtdIns4P levels were similar between Sac1 mutants and cells lacking the tethers, which suggests that the tether proteins facilitate PtdIns4P catalysis by Sac1.

Finally, the authors asked whether, in addition to influencing ER morphology, these tether proteins have a role in ER function. Although protein trafficking seemed unaffected, cells lacking the six tether proteins were hypersensitive to ER stress-inducing agents, which is indicative of an impaired unfolded protein response (UPR). Indeed, the UPR signalling pathway was active in mutant cells even in the absence of ER stress agents. Importantly, mutant cells showed impaired growth when UPR signalling was blocked, which indicates that upregulation of UPR signalling compensates for key ER functions that are lost in these mutant cells.

So, Ist2, Tcb1, Tcb2 and Tcb3, together with the previously described Scs2 and Scs22, seem to act as ER-plasma membrane tethers, facilitating cell signalling and promoting optimal ER function. As Scs proteins, Ist 2 and the tricalbins are conserved in higher eukaryotes, the authors propose that their function as tethers is also conserved.

Rachel David

ORIGINAL RESEARCH PAPER Manford, A. G. et al. ER-to-plasma membrane tethering proteins regulate cell signaling and ER morphology.

Dev. Cell 23, 1129-1140 (2012) 